\title{
Exceptional directions for Sierpiński's nonmeasurable sets
}

\author{
by
}

B. Kirchheim (Bratislava) and T. Natkaniec (Bydgoszcz)

\begin{abstract}
In [2] the question was considered in how many directions can a nonmeasurable plane set behave even "better" than the classical one constructed by Sierpiński in [6], in the sense that any line in a given direction intersects the set in at most one point. We considerably improve these results and give a much sharper estimate for the size of the sets of those "better" directions.
\end{abstract}

I. Let us establish some terminology to be used later. We shall denote by $\mathcal{L}$ the outer Lebesgue measure in the plane $\mathbb{R}^{2}$ and by $|\cdot|$ the outer Lebesgue measure on the real line $\mathbb{R}$. By $\ell(x, y)$ and $\ell_{\delta}(x)$ we denote the line through the two different points $x, y \in \mathbb{R}^{2}$ and the line through $x$ in the direction $\delta \in[0, \pi)$, respectively. For a given line $\ell \subset \mathbb{R}^{2}$ let $\operatorname{dir}(\ell) \in[0, \pi)$ be its direction. Further, for each $M \subset[0, \pi)$ let $\mathcal{C}_{M}$ denote the cone generated by $M$, i.e. $\mathcal{C}_{M}=\bigcup\left\{\ell_{\delta}(0): \delta \in M\right\}$. For $k \geq 1$ and $\delta \in[0, \pi)$ let $\mathcal{G}_{k}(\delta)$ be the class of all $E \subset \mathbb{R}^{2}$ such that for any line $\ell$ with $\operatorname{dir}(\ell)=\delta$ the set $E \cap \ell$ is open in $\ell$ and has at most $k$ connected components.

For any $M \subset \mathbb{R}$ we denote by $\operatorname{BSCL}(M)=M \cup\{x:(x-\varepsilon, x) \cap M \neq$ $\emptyset \neq(x, x+\varepsilon) \cap M$ for any $\varepsilon>0\}$, the "two-sided" closure of $M$. Finally, for $A \subset \mathbb{R}^{2}$ let $\operatorname{int}_{\mathrm{q}}(A)$ denote the interior of $A$ in the qualitative topology, i.e. the union of all subsets of $A$ of the form $U \backslash I$, where $U$ is open and $I$ is of the first category.

We shall use the following set-theoretical assumptions (see [3] for definitions). Let $A(m)$ and $U(m)$ stand for the propositions that the union of less than $\mathbf{c}$ (the continuum) measure zero sets has measure zero and that every set of reals of cardinality less than $\mathbf{c}$ has measure zero, respectively. Furthermore, let $A(c)$ and $U(c)$ be defined similarly with "first category" replacing measure zero. It is well known that all these conditions follow from Martin's Axiom and therefore also from the Continuum Hypothesis

1991 Mathematics Subject Classification: Primary 28A05. 
(see e.g. [5]). If not explicitly stated otherwise, we are working in ZFC without further assumptions.

A subset $S$ of $\mathbb{R}$ is called an $\mathcal{S}$-set (Sierpiński set) if $S \cap A$ has cardinality less than c for each $A \subset \mathbb{R}$ with Lebesgue measure zero. Note that every $\mathcal{S}$-set has inner measure zero and, under the assumption $A(m)$, it is easy to construct an $\mathcal{S}$-set having full outer measure (see e.g. [4]). A subset $L$ of $\mathbb{R}$ is called a Lusin set if $L \cap A$ has cardinality less than c for each nowhere dense $A \subset \mathbb{R}$.

II. Sierpiński [6] constructed an example of a nonmeasurable (and without the Baire property) subset $E$ of the plane whose intersection with any line consists of at most two points. It follows immediately that the complement of Sierpiński's set belongs to $\bigcap_{\delta \in[0, \pi)} \mathcal{G}_{3}(\delta)$ and therefore, we are mainly interested in the "better" classes $\mathcal{G}_{2}(\delta)$.

Frantz [2] proved that if $A(m)$ holds and $|[0, \pi) \backslash D|=0$ then every set in $\bigcap_{\delta \in D} \mathcal{G}_{2}(\delta)$ is measurable and, on the other hand, if $|D|=0$ then $\bigcap_{\delta \in D} \mathcal{G}_{2}(\delta) \cap \bigcap_{\delta \in[0, \pi)} \mathcal{G}_{3}(\delta)$ contains a nonmeasurable set. These results lead in a natural way to the following questions:

1. Is full outer measure of $D$ sufficient for measurability of sets in $\bigcap_{\delta \in D} \mathcal{G}_{2}(\delta) \cap \bigcap_{\delta \in[0, \pi)} \mathcal{G}_{3}(\delta) ?$

2. Is a positive inner measure of $D$ sufficient for measurability of sets in $\bigcap_{\delta \in D} \mathcal{G}_{2}(\delta) \cap \bigcap_{\delta \in[0, \pi)} \mathcal{G}_{3}(\delta) ?$

Proposition 1 below answers the first question in the negative.

LEMMA 1. Let $S \subset[0, \pi)$ be an $\mathcal{S}$-set. Then for each line $\ell \subset \mathbb{R}^{2}$ and for every $x \in \mathbb{R}^{2} \backslash \ell$ the set $\ell \cap \bigcup_{\delta \in S} \ell_{\delta}(x)$ is an $\mathcal{S}$-set on $\ell$.

Proof. Since every isometry maps $\mathcal{S}$-sets onto $\mathcal{S}$-sets, we can assume that $x=0$ and $\ell=\mathbb{R} \times\{1\}$. Let $h:(0, \pi) \rightarrow \ell$ be a homeomorphism defined by $h(x)=(\cot (x), 1)$ for $x \in(0, \pi)$. Then $h$ and $h^{-1}$ map measure zero sets onto measure zero sets and $\ell \cap \bigcup_{\delta \in S} \ell_{\delta}(0)=h(S \backslash\{0\})$. It is easy to verify that $h(S \backslash\{0\})$ is an $\mathcal{S}$-set and therefore $\ell \cap \bigcup_{\delta \in S} \ell_{\delta}(0)$ is an $\mathcal{S}$-set on $\ell$.

Proposition 1. Assume $A(m)$. Then for any $\mathcal{S}$-set $D \subset[0, \pi)$ the class $\bigcap_{\delta \in D} \mathcal{G}_{2}(\delta) \cap \bigcap_{\delta \in[0, \pi)} \mathcal{G}_{3}(\delta)$ contains nonmeasurable sets.

Proof. Let $\left(F_{\alpha}\right)_{\alpha<\mathbf{c}}$ be the net of all closed subsets of $\mathbb{R}^{2}$ with positive measure. Let $D \subset[0, \pi)$ be an $\mathcal{S}$-set. We choose inductively a net $\left(x_{\alpha}\right)_{\alpha<\mathbf{c}}$ such that

$$
x_{\alpha} \in F_{\alpha} \backslash\left(\left\{x_{\beta}: \beta<\alpha\right\} \cup \bigcup_{\beta, \gamma<\alpha} \ell\left(x_{\beta}, x_{\gamma}\right) \cup \bigcup_{\beta<\alpha}\left(x_{\beta}+\mathcal{C}_{D}\right)\right) .
$$

Assume that for some $\alpha<\mathbf{c}$ and for all $\beta<\alpha$ points $x_{\beta}$ are already defined. Fix $\delta \in[0, \pi) \backslash D$. According to Fubini's Theorem, there exists a line $\ell$ in 
the direction $\delta$ such that $x_{\beta} \notin \ell$ for $\beta<\alpha$ and $\ell \cap F_{\alpha}$ has a positive linear measure. Lemma 1 implies that $\ell \cap\left(x_{\beta}+\mathcal{C}_{D}\right)$ is an $\mathcal{S}$-set on $\ell$ for each $\beta<\alpha$. Since the union of less than $\mathbf{c} \mathcal{S}$-sets is an $\mathcal{S}$-set, the set

$$
\ell \cap\left(\bigcup_{\beta, \gamma<\alpha} \ell\left(x_{\beta}, x_{\gamma}\right) \cup \bigcup_{\beta<\alpha}\left(x_{\beta}+\mathcal{C}_{D}\right)\right)
$$

has linear inner measure zero and consequently,

$$
\ell \cap F_{\alpha} \backslash\left(\bigcup_{\beta, \gamma<\alpha} \ell\left(x_{\beta}, x_{\gamma}\right) \cup \bigcup_{\beta<\alpha}\left(x_{\beta}+\mathcal{C}_{D}\right)\right)
$$

is nonempty; select $x_{\alpha}$ from this set. Finally, it is easy to verify that the set $E=\mathbb{R}^{2} \backslash\left\{x_{\alpha}: \alpha<\mathbf{c}\right\}$ belongs to $\bigcap_{\delta \in D} \mathcal{G}_{2}(\delta) \cap \bigcap_{\delta \in[0, \pi)} \mathcal{G}_{3}(\delta)$ and is nonmeasurable.

Corollary 1. If we assume $A(m)$ then there exists $D \subset[0, \pi)$ of full outer measure for which $\bigcap_{\delta \in D} \mathcal{G}_{2}(\delta) \cap \bigcap_{\delta \in[0, \pi)} \mathcal{G}_{3}(\delta)$ contains nonmeasurable sets.

The next theorem answers our second question in the affirmative.

TheOREM 1. Let $D \subset[0, \pi)$ have positive inner measure. Then any $E \in \bigcap_{\delta \in D} \mathcal{G}_{2}(\delta)$ is measurable.

This theorem is a special case of the following proposition (set $n=1$ and $\left.c=|D|_{*} / 2 \pi\right)$, which will be applied below a second time and which uses an idea from Theorem 2 in [2].

Proposition 2. Let $c>0, n \geq 1$ and $D \subset[0, \pi)$ be such that for all $x^{1}, \ldots, x^{n} \in \mathbb{R}^{2}$ mutually different and any $j \leq n$

$$
\limsup _{r \searrow 0} \frac{\mathcal{L}_{*}\left(\left(\bigcup_{i=1}^{n}\left(x^{i}+\mathcal{C}_{D}\right)\right) \cap B\left(x^{j}, r\right)\right)}{\mathcal{L}\left(B\left(x^{j}, r\right)\right)}>c,
$$

where $B(x, r)=\left\{y \in \mathbb{R}^{2}:\|x-y\|<r\right\}$ and $\mathcal{L}_{*}(A)$ denotes the inner measure of $A$. Then each $E \in \bigcap_{\delta \in D} \mathcal{G}_{2}(\delta)$ is Lebesgue measurable.

Proof. Using a suitable rotation of the plane, we may assume that $0 \in$ $D$. For any $p<q$ let $E(p, q)=\{y:(p, q) \times\{y\} \subset E\}$. Obviously, $E \in \mathcal{G}_{2}(0)$ implies $E=\bigcup\{(p, q) \times E(p, q): p<q$ rationals $\}$. Hence, the measurability will follow if we show that for any $p<q$ there exists a measurable set $A$ satisfying $(p, q) \times E(p, q) \subset A \subset E$. But this is a consequence of the following statement:

$$
\text { for any } p<q, \mathcal{L}(((p, q) \times \operatorname{BSCL}(E(p, q))) \backslash E)=0 \text {. }
$$

Indeed, since $\mathbb{R} \backslash \operatorname{BSCL}(E(p, q))$ has all connected components nondegenerate and hence at most countably many of them, we infer that $(p, q) \times$ 
$\operatorname{BSCL}(E(p, q))$ is a $G_{\delta}$-set, and hence measurable. Therefore, $(*)$ ensures that we could choose

$$
A=((p, q) \times \operatorname{BSCL}(E(p, q))) \backslash((p, q) \times \operatorname{BSCL}(E(p, q)) \backslash E) .
$$

So, let us prove $(*)$. Assume

$$
S=((p, q) \times \operatorname{BSCL}(E(p, q))) \backslash E
$$

is not of measure zero and let $\widetilde{S}$ be a measurable hull of $S$. Lebesgue's density theorem (see e.g. [1], p. 184) implies that there exists a measure zero set $S_{0}$ such that any $x \in \widetilde{S} \backslash S_{0}$ is a density point of $\widetilde{S}$ and hence a point of outer density of $S$. Consequently, we can find $x^{1} \in S \backslash S_{0}$. Obviously

$$
x_{1}^{1} \in(p, q) \quad \text { and } \quad x_{2}^{1} \in \operatorname{BSCL}(E(p, q)) \backslash E(p, q),
$$

therefore we can choose $s, t \in E(p, q)$ with $s<x_{2}^{1}<t$ such that

$$
\mathcal{L}\left(B\left(x^{1}, 1\right) \cap L_{x^{1}}\right)>(1-c) \mathcal{L}\left(B\left(x^{1}, 1\right)\right)
$$

where $L_{y}=\bigcup\left\{\ell_{\delta}(y): \ell_{\delta}(y) \cap((p, q) \times\{s\}) \neq \emptyset\right.$ and $\left.\ell_{\delta}(y) \cap((p, q) \times\{t\}) \neq \emptyset\right\}$ for general $y$. This inequality together with the definition of a point of outer density imply that we can find $n-1$ different points $x^{2}, \ldots, x^{n} \in$ $S \cap((p, q) \times(s, t)) \cap L_{x^{1}} \backslash\left\{x^{1}\right\}$. Since $L_{y} \backslash\{y\}$ is always open, there is an $R>0$ such that

$$
B\left(x^{1}, R\right) \subset((p, q) \times(s, t)) \cap L_{x^{2}} \cap \ldots \cap L_{x^{n}} \backslash\left\{x^{2}, \ldots, x^{n}\right\} .
$$

Now notice that for any $x \in S \cap((p, q) \times(s, t))$,

$$
L_{x} \cap\left(x+\mathcal{C}_{D}\right) \cap((p, q) \times(s, t)) \backslash\{x\} \subset E .
$$

Indeed, by definition any line $\ell_{\delta}(x)$ contained in $L_{x} \cap\left(x+\mathcal{C}_{D}\right)$ intersects $(p, q) \times\{s, t\} \subset E$ on both sides of $x$ and has on both sides of $x$ precisely one connected component of $\ell_{\delta}(x) \cap E$ (since $x \notin E$ and $\delta \in D$ ). However, since $\delta \neq 0$ and since $x_{2}^{1}$ is a two-sided accumulation point of $E(p, q)$, the union of those two connected components also contains $\ell_{\delta}(x) \cap((p, q) \times(s, t)) \backslash\{x\}$, as was to be shown.

Summarizing we obtain

$$
\left[\left(x^{1}+\mathcal{C}_{D}\right) \cup \ldots \cup\left(x^{n}+\mathcal{C}_{D}\right)\right] \cap B\left(x^{1}, R\right) \cap L_{x^{1}} \backslash\left\{x^{1}\right\} \subset E .
$$

However, according to the assumptions on $D$ and $L_{x^{1}}$,

$$
\limsup _{r \searrow 0} \frac{\mathcal{L}_{*}\left(E \cap B\left(x^{1}, r\right)\right)}{\mathcal{L}\left(B\left(x^{1}, r\right)\right)}>0 .
$$

But, since $E \cap S=\emptyset$, this contradicts the fact that $x^{1}$ is a point of outer density of $S$ and proves $(*)$ as well as the whole proposition.

According to Proposition 1, outer measure cannot be used to guarantee that a given set $D \subset[0, \pi)$ is sufficiently large to make each $E \in \bigcap_{\delta \in D} \mathcal{G}_{2}(\delta)$ measurable. Since the set constructed there was of inner measure zero, 
this example together with Theorem 1 could lead to the conjecture that $\bigcap_{\delta \in D} \mathcal{G}_{2}(\delta)$ consists only of measurable sets iff $D \subset[0, \pi)$ has positive inner measure. However, this is refuted by

TheOREM 2. Assume $U(m)$. Then there exists $D \subset[0, \pi)$ of inner measure zero such that any $E \in \bigcap_{\delta \in D} \mathcal{G}_{2}(\delta)$ is measurable.

Indeed, this is an immediate consequence of Proposition 2 and the following

Proposition 3. There exists $M \subset[0, \pi)$ with no nonempty perfect subset and such that

$$
V=\mathbb{R}^{2} \backslash\left[\left(x+\mathcal{C}_{M}\right) \cup\left(y+\mathcal{C}_{M}\right) \cup\left(z+\mathcal{C}_{M}\right)\right]
$$

is contained in the union of less than $\mathbf{c}$ lines whenever $x, y, z \in \mathbb{R}^{2}$ are three different points. Hence $M$ has inner measure zero, $\operatorname{int}_{\mathrm{q}}(M)=\emptyset$ and if we suppose $U(m)$ (resp. $U(c)$ ) to be true then $V$ has measure zero (resp. is of the first category) whenever $x, y, z$ are different.

Proof. For any $s, t \in[0, \pi)$ and any three different $x, y, z \in \mathbb{R}^{2}$ we set $T(x, y, z)=\ell(x, y) \cup \ell(y, z) \cup \ell(z, x)$ and let $M(s, t ; x, y, z)$ be the set of all $u \in$ $[0, \pi)$ such that there is a bijection $f:\{1,2,3\} \rightarrow\{x, y, z\}$ with $\ell_{s}(f(1)) \cap$ $\ell_{t}(f(2)) \cap \ell_{u}(f(3)) \backslash T(x, y, z) \neq \emptyset$. Simple geometrical reasoning yields that for any fixed $f$ there is at most one such $u$ and hence $M(s, t ; x, y, z)$ contains at most 6 elements. Next, let $\left\{C_{\alpha}\right\}_{\alpha<\mathbf{c}}$ be the system of all perfect subsets of $[0, \pi)$ and let $\left\{\left(x^{\alpha}, y^{\alpha}, z^{\alpha}\right)\right\}_{\alpha<\mathbf{c}}$ be an enumeration of all triples of different points in $\mathbb{R}^{2}$.

Now, assume that for some $\kappa<\mathbf{c}$ all $t_{\alpha}, \alpha<\kappa$, have already been chosen. Then a simple comparison of cardinalities shows that we can select some

$$
t_{\kappa} \in C_{\kappa} \backslash \bigcup_{\alpha \leq \kappa} \bigcup_{\beta, \gamma<\kappa} M\left(t_{\beta}, t_{\gamma} ; x^{\alpha}, y^{\alpha}, z^{\alpha}\right) .
$$

Finally, the set $M=[0, \pi) \backslash\left\{t_{\alpha}: \alpha<\mathbf{c}\right\}$ has all the required properties. From $(*)$ it is obvious that $M$ contains no nonempty perfect sets. To verify the second property of $M$ it suffices to show that for each $\kappa<\mathbf{c}$ the set

$$
S_{\kappa}=\left(x^{\kappa}+\mathcal{C}_{[0, \pi) \backslash M}\right) \cap\left(y^{\kappa}+\mathcal{C}_{[0, \pi) \backslash M}\right) \cap\left(z^{\kappa}+\mathcal{C}_{[0, \pi) \backslash M}\right)
$$

is a subset of

$$
T\left(x^{\kappa}, y^{\kappa}, z^{\kappa}\right) \cup\left(x^{\kappa}+\mathcal{C}_{\left\{t_{\alpha}: \alpha<\kappa\right\}}\right) \cup\left(y^{\kappa}+\mathcal{C}_{\left\{t_{\alpha}: \alpha<\kappa\right\}}\right) \cup\left(z^{\kappa}+\mathcal{C}_{\left\{t_{\alpha}: \alpha<\kappa\right\}}\right) .
$$

(Note that if we assume $U(m)$ (resp. $U(c)$ ) then this last set has measure zero (resp. is of the first category).)

But indeed, if $p \in S_{\kappa} \backslash T\left(x^{\kappa}, y^{\kappa}, z^{\kappa}\right)$ then $p \in \ell_{t_{\alpha}}\left(x^{\kappa}\right) \cap \ell_{t_{\beta}}\left(y^{\kappa}\right) \cap \ell_{t_{\gamma}}\left(z^{\kappa}\right)$ for three different ordinals $\alpha, \beta, \gamma<\mathbf{c}$. Hence there are $\alpha^{\prime}<\beta^{\prime}<\gamma^{\prime}<\mathbf{c}$ with 
$\{\alpha, \beta, \gamma\}=\left\{\alpha^{\prime}, \beta^{\prime}, \gamma^{\prime}\right\}$ and of course, $t_{\gamma^{\prime}} \in M\left(t_{\alpha^{\prime}}, t_{\beta^{\prime}} ; x^{\kappa}, y^{\kappa}, z^{\kappa}\right)$. According to $(*)$ this implies $\gamma^{\prime}<\kappa$ and

$$
p \in\left(x^{\kappa}+\mathcal{C}_{\left\{t_{\alpha}: \alpha<\kappa\right\}}\right) \cup\left(y^{\kappa}+\mathcal{C}_{\left\{t_{\alpha}: \alpha<\kappa\right\}}\right) \cup\left(z^{\kappa}+\mathcal{C}_{\left\{t_{\alpha}: \alpha<\kappa\right\}}\right) .
$$

Let us remark that in the same way as Proposition 3 we can prove in ZFC that for any three different points $x, y, z$ there exists a set $M$ of inner measure zero such that

$$
\left(x+\mathcal{C}_{M}\right) \cup\left(y+\mathcal{C}_{M}\right) \cup\left(z+\mathcal{C}_{M}\right)=\mathbb{R}^{2} .
$$

On the other hand, it is easy to observe that there is no "universal" set $M$ of inner measure zero which has the above property for each triple $x, y, z$ of distinct points in $\mathbb{R}^{2}$.

III. The well-known correlation between measure and category [4] suggests that category analogues of the foregoing results are true. Indeed, one can prove the following theorems (we omit the proofs which are quite the same as in the second section).

Proposition 4. Assume $A(c)$. If $D \subset[0, \pi)$ is of the first category then there exists a set $E$ in $\bigcap_{\delta \in D} \mathcal{G}_{2}(\delta) \cap \bigcap_{\delta \in[0, \pi)} \mathcal{G}_{3}(\delta)$ without the Baire property.

Proposition 5. Assume $A(c)$. Then there exists $D \subset[0, \pi)$ which is of the second category at each point of $[0, \pi)$ and for which $\bigcap_{\delta \in D} \mathcal{G}_{2}(\delta) \cap$ $\bigcap_{\delta \in[0, \pi)} \mathcal{G}_{3}(\delta)$ contains sets without the Baire property.

Proposition 6. Let $0<a<b<\pi, n \geq 1$ and $D \subset[0, \pi)$ satisfy

$$
\left\{x^{1}, \ldots, x^{n}\right\}+\mathcal{C}_{(a, b)} \subset \operatorname{cl}\left(\operatorname{int}_{\mathrm{q}}\left(\bigcup_{i=1}^{n}\left(x^{i}+\mathcal{C}_{D}\right)\right)\right)
$$

for any different points $x^{1}, \ldots, x^{n} \in \mathbb{R}^{2}$. Then each set $E$ in $\bigcap_{\delta \in D} \mathcal{G}_{2}(\delta)$ has the Baire property.

Proof. Similarly to the proof of Proposition 2 it is enough to prove that for each pair of rationals $p, q$ the set $S=((p, q) \times \operatorname{BSCL}(E(p, q))) \backslash E$ is of the first category. Suppose that $S$ is of the second category. Then there exist nonempty intervals $U \subset(p, q)$ and $V$ such that $S$ is of the second category at each point of $U \times V$. Fix $x^{1} \in S \cap(U \times V)$ and $s<x_{2}^{1}<t$ with $s, t \in V \cap E(p, q)$ and such that the open set $\left\{\delta \in(a, b): \ell_{\delta}\left(x^{1}\right) \subset L_{x^{1}}\right\}$ is nonvoid. In the same way as in the proof of Proposition 2 we can choose $n-1$ different points $x^{2}, \ldots, x^{n} \in S \cap(U \times(s, t)) \cap L_{x^{1}} \backslash\left\{x^{1}\right\}$ and $R>0$ such that

$$
B\left(x^{1}, R\right) \subset(U \times V) \cap L_{x^{2}} \cap \ldots \cap L_{x^{n}} \backslash\left\{x^{2}, \ldots, x^{n}\right\}
$$


and

$$
\left[\left(x^{1}+\mathcal{C}_{D}\right) \cup \ldots \cup\left(x^{n}+\mathcal{C}_{D}\right)\right] \cap B\left(x^{1}, R\right) \cap L_{x^{1}} \backslash\left\{x^{1}\right\} \subset E .
$$

From the assumption on $D$ it follows that $E \cap B\left(x^{1}, R\right)$ contains a second category subset with the Baire property, which contradicts the assumption on $U \times V$.

THEOREM 3. If $D \subset[0, \pi)$ contains a second category set having the Baire property, then each set in $\bigcap_{\delta \in D} \mathcal{G}_{2}(\delta)$ also has the Baire property.

Proposition 7. Assume $U(c)$. Then there exists $M \subset[0, \pi)$ such that $\operatorname{int}_{\mathrm{q}}(M)=\emptyset$ and $\mathbb{R}^{2} \backslash\left[\left(x+\mathcal{C}_{M}\right) \cup\left(y+\mathcal{C}_{M}\right) \cup\left(z+\mathcal{C}_{M}\right)\right]$ is of the first category whenever $x, y, z$ are three different points of $\mathbb{R}^{2}$.

TheOREM 4. Assume $U(c)$. Then there exists $D \subset[0, \pi)$ such that $\operatorname{int}_{\mathrm{q}}(D)=\emptyset$ and any set in $\bigcap_{\delta \in D} \mathcal{G}_{2}(\delta)$ has the Baire property.

IV. Now we try to find conditions for $\bigcap_{\delta \in[0, \pi)} \mathcal{G}_{3}(\delta) \cap \bigcap_{\delta \in D} \mathcal{G}_{2}(\delta)$ to contain non-Borel sets.

First, Frantz [2] showed that $\bigcap_{\delta \in[0, \pi)} \mathcal{G}_{3}(\delta) \cap \bigcap_{\delta \in D} \mathcal{G}_{2}(\delta)$ contains only open sets provided that $[0, \pi) \backslash D$ is finite.

Theorem 5. If $[0, \pi) \backslash D$ is countable then any set in $\bigcap_{\delta \in[0, \pi)} \mathcal{G}_{3}(\delta) \cap$ $\bigcap_{\delta \in D} \mathcal{G}_{2}(\delta)$ is of type $G_{\delta \sigma}$.

Proof. Let $E \in \bigcap_{\delta \in[0, \pi)} \mathcal{G}_{3}(\delta) \cap \bigcap_{\delta \in D} \mathcal{G}_{2}(\delta)$. We may assume that $0, \pi / 2 \in D$. For $p<q$ define

$$
E(p, q)=\{y:(p, q) \times\{y\} \subset E\}, \quad E^{*}(p, q)=\{x:\{x\} \times(p, q) \subset E\} .
$$

Obviously, $E \in \mathcal{G}_{2}(0) \cap \mathcal{G}_{2}(\pi / 2)$ implies

$$
\begin{aligned}
E=\bigcup\left\{[(p, q) \times E(p, q)] \cap\left[E^{*}\left(p^{\prime}, q^{\prime}\right) \times\left(p^{\prime}, q^{\prime}\right)\right]:\right. & \\
& \left.p<q, p^{\prime}<q^{\prime} \text { rationals }\right\} .
\end{aligned}
$$

Similarly to the proof of Proposition 2 it is enough to verify that for any $p<q$ and $p^{\prime}<q^{\prime}$ the set

$$
M=[(p, q) \times \operatorname{BSCL}(E(p, q))] \cap\left[\operatorname{BSCL}\left(E^{*}\left(p^{\prime}, q^{\prime}\right)\right) \times\left(p^{\prime}, q^{\prime}\right)\right] \backslash E
$$

is countable. One easily observes that for each line $\ell$ any $x \in \ell \cap M$ is a twosided cluster point of $\ell \cap E$. Hence, for a fixed $x_{0} \in M$ the set $\ell_{\delta}\left(x_{0}\right) \cap M$ contains at most two points and moreover, $\ell_{\delta}\left(x_{0}\right) \cap M=\left\{x_{0}\right\}$ whenever $\delta \in D$. Since $[0, \pi) \backslash D$ is countable, the proof is finished.

But what happens for general $D \subset[0, \pi)$ ? First assume that $\operatorname{card}([0, \pi) \backslash$ $D)<$ c. If we assume $\mathrm{CH}$ then from the foregoing it follows that every set in $\bigcap_{\delta \in[0, \pi)} \mathcal{G}_{3}(\delta) \cap \bigcap_{\delta \in D} \mathcal{G}_{2}(\delta)$ is Borel measurable. On the other hand, if we assume non- $\mathrm{CH}$ then there exists $D \subset[0, \pi)$ such that $\operatorname{card}([0, \pi) \backslash D)<\mathbf{c}$ 
and $\bigcap_{\delta \in[0, \pi)} \mathcal{G}_{3}(\delta) \cap \bigcap_{\delta \in D} \mathcal{G}_{2}(\delta)$ contains non-Borel sets. Indeed, let $M \subset \mathbb{R}^{2}$ be a set having no three points on a line and with cardinality strictly between $\aleph_{0}$ and c. Then $D=[0, \pi) \backslash\{\operatorname{dir}(\ell(x, y)): x, y \in M, x \neq y\}$ has the mentioned properties.

Finally, we allow $[0, \pi) \backslash D$ of cardinality $\mathbf{c}$.

Proposition 8. There exists an open set $D \subset[0, \pi)$ of full measure such that $\bigcap_{\delta \in[0, \pi)} \mathcal{G}_{3}(\delta) \cap \bigcap_{\delta \in D} \mathcal{G}_{2}(\delta)$ contains $2^{\mathbf{c}}$ nonprojective sets.

Proof. Let $M \subset[0, \pi / 2)$ be a perfect set with symmetrical index (also called packing dimension) $\operatorname{Dim}(E)=0$ (see [7]). Then the sets $\widetilde{M}$ of all points of $\mathbb{R}^{2}$ with polar coordinates $r=1$ and $\phi \in M$ and $D=[0, \pi) \backslash[\pi / 2+$ $(1 / 2)(M+M)]($ where $\pi / 2+(1 / 2)(M+M)=\{\pi / 2+(1 / 2)(y+z): y, z \in M\})$ are perfect and have packing dimension zero [7]. Consequently, $D$ is open and has full measure. Let $E$ be a fixed subset of $\widetilde{M}$. Evidently, $E^{c} \in$ $\bigcap_{\delta \in[0, \pi)} \mathcal{G}_{3}(\delta)$ where $E^{c}=\mathbb{R}^{2} \backslash E$. We shall verify that $E^{c} \in \bigcap_{\delta \in D} \mathcal{G}_{2}(\delta)$. Assume that $E^{c} \notin \mathcal{G}_{2}(\gamma)$ for some $\gamma \in[0, \pi)$. Then there exist $x, y \in \widetilde{M}$ for which the line $\ell(x, y)$ has direction $\gamma$. Let $x$ and $y$ have polar coordinates $(1, \alpha)$ and $(1, \beta)$, respectively. Then simple geometrical reasoning yields that $\gamma=\pi / 2+(1 / 2)(\alpha+\beta) \in \pi / 2+(1 / 2)(M+M)$ and therefore, $\gamma \notin D$. Obviously, there are $2^{\mathbf{c}}$ nonprojective subsets of $\widetilde{M}$.

Theorem 6. For $D \subset[0, \pi)$ the family $\bigcap_{\delta \in[0, \pi)} \mathcal{G}_{3}(\delta) \cap \bigcap_{\delta \in D} \mathcal{G}_{2}(\delta)$ contains a non-Borel set iff

(*) there is an uncountable set $M \subset \mathbb{R}^{2}$ having no three points on any line and no two points on lines with directions in $D$.

Proof. Obviously, the condition $(*)$ is sufficient. Indeed, if $M$ fulfills (*), then there exists a non-Borel subset $E$ of $M$ with $E \in \bigcap_{\delta \in[0, \pi)} \mathcal{G}_{3}(\delta) \cap$ $\bigcap_{\delta \in D} \mathcal{G}_{2}(\delta)$. Now assume that $\bigcap_{\delta \in[0, \pi)} \mathcal{G}_{3}(\delta) \cap \bigcap_{\delta \in D} \mathcal{G}_{2}(\delta)$ contains a nonBorel set $E$. Let $\delta_{1}, \delta_{2}$ be two different directions in $D$; assume for convenience that $\delta_{1}=0$ and $\delta_{2}=\pi / 2$. For $p, q, p^{\prime}, q^{\prime}$ let

$$
\begin{aligned}
E_{p, q, p^{\prime}, q^{\prime}} & =[(p, q) \times \operatorname{BSCL}(E(p, q))] \cap\left[\operatorname{BSCL}\left(E^{*}\left(p^{\prime}, q^{\prime}\right)\right) \times\left(p^{\prime}, q^{\prime}\right)\right], \\
M_{p, q, p^{\prime}, q^{\prime}} & =E_{p, q, p^{\prime}, q^{\prime}} \backslash E .
\end{aligned}
$$

Since

$$
E=\bigcup\left\{E_{p, q, p^{\prime}, q^{\prime}} \backslash M_{p, q, p^{\prime}, q^{\prime}}: p<q, p^{\prime}<q^{\prime} \text { rationals }\right\}
$$

and the $E_{p, q, p^{\prime}, q^{\prime}}$ are $G_{\delta}$-sets, there are $p<q$ and $p^{\prime}<q^{\prime}$ such that $M=$ $M_{p, q, p^{\prime}, q^{\prime}}$ is uncountable. As in the proof of Theorem 5 we observe that for any line $\ell$ the set $\ell \cap M$ has at most two points and is a singleton whenever $\operatorname{dir}(\ell) \in D$. Thus $M$ satisfies $(*)$. 
Corollary 2. Assume $C H$. Then the set $M$ defined in Proposition 3 is non-Borel (since it is nonmeasurable) and does not satisfy the condition $(*)$. Hence any set in $\bigcap_{\delta \in[0, \pi)} \mathcal{G}_{3}(\delta) \cap \bigcap_{\delta \in M} \mathcal{G}_{2}(\delta)$ is Borel measurable.

\section{References}

[1] D. L. Cohn, Measure Theory, Birkhäuser, 1980.

[2] M. Frantz, On Sierpiński's nonmeasurable set, Fund. Math. 139 (1991), 17-22.

[3] A. Miller, Some properties of measure and category, Trans. Amer. Math. Soc. 266 (1981), 93-114.

[4] J. Oxtoby, Measure and Category, Springer, 1971.

[5] J. Shoenfield, Martin's Axiom, Amer. Math. Monthly 82 (1975), 610-617.

[6] W. Sierpiński, Sur un problème concernant les ensembles mesurables superficiellement, Fund. Math. 1 (1920), 112-115.

[7] U. Tricot, Two definitions of fractional dimension, Math. Proc. Cambridge Philos. Soc. 91 (1982), 57-75.

INSTITUTE OF APPLIED MATHEMATICS

INSTITUTE OF MATHEMATICS, WSP COMENIUS UNIVERSITY

MLYNSKÁ DOLINA

CHODKIEWICZA 30

84215 BRATISLAVA, CZECHOSLOVAKIA

85-064 BYDGOSZCZ, POLAND 